\title{
PROXIMINAL SUBSPACES Of $A(K)$ Of FINITE CODIMENSION
}

\author{
T. S. S. R. K. RAO
}

Received 30 December 2002

\begin{abstract}
We study an analogue of Garkavi's result on proximinal subspaces of $C(X)$ of finite codimension in the context of the space $A(K)$ of affine continuous functions on a compact convex set $K$. We give an example to show that a simple-minded analogue of Garkavi's result fails for these spaces. When $K$ is a metrizable Choquet simplex, we give a necessary and sufficient condition for a boundary measure to attain its norm on $A(K)$. We also exhibit proximinal subspaces of finite codimension of $A(K)$ when the measures are supported on a compact subset of the extreme boundary.
\end{abstract}

2000 Mathematics Subject Classification: 41A50, 46B20.

1. Introduction. This note is motivated by a well-known result of Garkavi (see [5, page 302]) that characterizes proximinal subspaces of finite codimension in $C(X)$, the space of continuous functions on a compact Hausdorff space $X$. See $[2,6]$ for other recent characterizations of proximinal subspaces of finite codimension in $C(X)$ that are modelled on Garkavi's result. In this short note, we study proximinal subspaces of finite codimension in the space $A(K)$ of affine continuous functions (over the real scalar field) on a metrizable Choquet simplex $K$. For a compact convex set $K$, let $\partial K$ denote the set of extreme points of $K$. We recall from [1] that a metrizable compact convex set $K$ is a Choquet simplex if for every $k \in K$, there exists a unique regular Borel probability measure $\mu$ such that $\mu(\partial K)=1$ and $\int a d \mu=a(k)$ for all $a \in A(K)(k$ is said to be the resultant of $\mu$ ). It is well known in the literature that for a simplex $K$, the space $A(K)$ shares several properties of the space of continuous functions (see [1]). In particular, for any simplex $K$ whose set of extreme points $\partial K$ is closed, $A(K)$ is isometric to $C(\partial K)$ (see [1, Theorem II.4.3]). Thus it is natural to look for an analogue of Garkavi's theorem in this context. As far as we are aware, this is the first time that such an analogue is being attempted for subspaces of the space of continuous functions.

We first consider subspaces of codimension 1. Since a hyperplane is proximinal if and only if the defining continuous linear functional attains its norm, we first attempt to describe norm attaining functional of $A(K)^{*}$. Given $\Phi \in A(K)^{*}$, one can consider a norm-preserving Hahn-Banach extension $\Phi^{\prime} \in C(K)^{*}$, and since if $\Phi$ attains its norm on $A(K), \Phi^{\prime}$ attains its norm on $C(K)$, one can try 
to apply Garkavi's result. In general, as the extension is not unique, this procedure does not give much information about $\Phi$. However, if one restricts the extension to the so-called boundary measures (we identify $C(K)^{*}$ via the Riesz representation theorem with the space of regular Borel measures $M(K)$ on $K)$, it follows from [1, Lemma II.3.5] that the restriction map $\beta: C(K)^{*} \rightarrow A(K)^{*}$ is one-to-one and onto on the space of boundary measures. Thus it is reasonable to investigate analogues of Garkavi's result for subspaces of finite codimension described by boundary measures.

We start with an example of a simplex space and subspaces of codimension 1 and 2 described by boundary measures that are not proximinal but the corresponding subspaces in the spaces of continuous functions are proximinal. For a metrizable Choquet simplex $K$, we give a necessary and sufficient condition for a boundary measure to attain its norm on $A(K)$ extending Garkavi's result in this case. We refer to $[1,4]$ for all the concepts of convexity theory that we will be using here.

MAIN RESUlt. Let $X$ be a compact Hausdorff space. For a $\mu \in C(X)^{*}$, let supp $\mu$ denote the smallest closed subset of $X$ such that $|\mu|(\operatorname{supp} \mu)=\|\mu\|$. Let $G \subset C(X)$ be a subspace of codimension $n$. Suppose $G^{\perp}=\operatorname{span}\left\{\mu_{1}, \ldots, \mu_{n}\right\}$. We first recall Garkavi's result [5, Theorem 2.2, page 302].

THEOREM 1.1 (Garkavi). In order that $G$ be proximinal, it is necessary and sufficient that the following three conditions be satisfied.

(1) For every $0 \neq \mu \in G^{\perp}$, supp $\mu$ admits a Hahn decomposition into two closed sets $\operatorname{supp} \mu^{+}$and $\operatorname{supp} \mu^{-}=\operatorname{supp} \mu \backslash \operatorname{supp} \mu^{+}$.

(2) For every pair of nonzero measures $\mu$ and $v$ in $G^{\perp}$, the set $\operatorname{supp} \mu \backslash \operatorname{supp} v$ is closed.

(3) For every pair of nonzero measures $\mu$ and $\nu$ in $G^{\perp}$, the measure $\mu$ is absolutely continuous with respect to the measure $v$ on the set $\operatorname{supp} \mu$.

We recall from [1] that when $K$ is metrizable, $\partial K$ is a $G_{\delta}$ set and boundary measures are precisely those measures $\mu$ for which $|\mu|(\partial K)=\|\mu\|$. For a Banach space $Y$, we denote by $Y_{1}$ its closed unit ball.

The following example is to show that a simple-minded analogue of Garkavi's theorem is not valid in the case of $A(K)$. We first look at the case of codimension 1 . Thus only condition (1) of the above theorem need to be considered. In what follows, we will be using the Hahn decomposition of the support of a norm attaining measure given by the above theorem and the structure of the sets that is contained in the proof of the theorem as given in [5].

EXAMPLE 1.2. Let $A=\left\{f \in C(\mathbb{N} \cup\{\infty\}): f(\infty)=\sum\left(1 / 2^{n}\right) f(n)\right\}$, where $\mathbb{N} \cup$ $\{\infty\}$ is the one-point compactification of $\mathbb{N}$. Then $A$ is an $A(K)$ space where $K$ is the state space of $A$ with $\partial K=\mathbb{N}$ and it can be easily seen that $K$ is a metrizable Choquet simplex. Note that if a measure $\mu \in A^{*}$ attains its norm on $A$, then it attains it on the continuous function space and hence, if $\mu$ is supported on 
an infinite subset $\mathbf{A}$ of $\mathbb{N}$, Garkavi's theorem gives a Hahn decomposition of $\mathbf{A} \cup\{\infty\}=B \cup C$, disjoint closed sets, say, $C$ finite, $B$ infinite containing $\infty$.

It is easy to see that a finitely supported boundary measure $\mu$ attains its norm on $A$ if and only if it attains its norm on the continuous function space $C(\mathbb{N} \cup\{\infty\})$.

Now we will show that if a boundary measure $\mu \in A^{*}$, supported on an infinite subset of $\mathbb{N}$, attains its norm on $A$, then $\mu$ is either a positive or negative measure, that is, one of the sets in Garkavi's decomposition is empty. Thus one can easily give examples (see below) of boundary measures that attain their norm on the continuous function space but not on $A$.

Let $\mu=\sum \alpha_{i} \delta(i)$ be such that each $\alpha_{i} \neq 0$ and $\sum\left|\alpha_{i}\right|=1$ (same proof works if infinitely many $\alpha_{i}$ 's are nonzero). If $\|f\|=1=\sum \alpha_{i} f(i)$, then $|f(i)|=1$ for every $i$, and since $f \in A, f$ is identically 1 or -1 . Thus $\mu$ is positive or negative.

We further note that if $\mu$ and $v$ are two independent boundary measures attaining their norm, if one of them is infinitely supported, we can choose an $\alpha$ so that $\mu+\alpha v$ takes both positive and negative values. Since for any subspace $M$ of finite codimension, every vector in $M^{\perp}$ attains its norm (see [5, Lemma III.1.1, page 292]), we conclude that $M$ cannot have measures of the above kind.

As an illustration, take $\mu=\sum\left(1 / 2^{n}\right) \delta(n+1)$ and $\nu=\delta(1)$. Since $\mu$ and $\nu$ are mutually singular, for any $\alpha$, it is easy to see that $\mu+\alpha \nu$ attains its norm on $C(\mathbb{N} \cup\{\infty\})$ but $\mu-v$ does not attain its norm on $A$.

Let $\phi=\delta(\infty)$, which is not a boundary measure for $A$. It is easy to see that $\operatorname{ker}(\mu) \cap \operatorname{ker}(\nu)=\operatorname{ker}(\phi) \cap \operatorname{ker}(\nu)$. Thus this subspace of codimension 2 in $A$ contains two independent boundary measures of the above form, hence is not a proximinal subspace. As $\mu$ and $v$ are mutually singular measures, one can show that the three conditions of the theorem of Garkavi are satisfied, and hence the intersections of their kernels is a proximinal subspace of $C(\mathbb{N} \cup\{\infty\})$.

REMARK 1.3. For a general Choquet simplex $K$, if the support of a measure $\mu$ is a compact subset of $\partial K$, then $\mu$ attains its norm on $A(K)$ if and only if it attains its norm on $C(K)$.

This follows from the general Choquet theory. Let $S=\operatorname{supp} \mu$. If $\mu$ attains its norm on $C(K)$, we, in particular, have an $f \in C(S)_{1}$ such that $\mu(f)=\|\mu\|$. But any such $f$ has a norm-preserving extension to $A(K)$ (see [1, Theorem II.3.12]). Thus $\mu$ attains its norm on $A(K)$.

This idea can now be used to give a general formulation for a measure to attain its norm on $A(K)$ for any compact convex set $K$.

THEOREM 1.4. Let $\mu$ be a measure on $K$ of norm one. Suppose supp $\mu=A \cup B$ for disjoint closed sets $A, B$, which is a Hahn decomposition, and further, their closed convex hulls are split faces of $K$, then $\mu$ attains its norm on $A(K)$. Conversely, suppose $K$ is a metrizable Choquet simplex, $\mu$ is a boundary measure, and the closed convex hull of supp $\mu$ is a face. Then if $\mu$ attains its norm, $\operatorname{supp} \mu$ has a decomposition of the above form. 
Proof. Suppose we have such decomposition. Let $F=\mathrm{CO}^{-}(A)$ and $G=$ $\mathrm{CO}^{-}(B)$. Note that they are disjoint. This is because since $F \cap G$ is a closed face, we only need to observe that it has no extreme points of $K$.

Indeed if $x \in F \cap G$ is an extreme point, then, by Milman's theorem (see [4, Proposition 1.5]), $x \in A \cap B$. Thus $F$ and $G$ are disjoint.

Also note that $\mathrm{CO}(F \cup G)$ is a split face (see [1, Corollary II.6.8]) and $G$ is the complementary face of $F$ relative to the set $\operatorname{CO}(F \cup G)$. Thus there exists an $a \in A(\operatorname{CO}(F \cup G))_{1}$ such that $\mu(a)=1$ (see [1, Proposition II.6.19]). Since $\mathrm{CO}(F \cup G)$ is a split face, this $a$ has a norm-preserving extension to $a^{\prime} \in A(K)$ (see [1, Theorem II.6.15]) and $\mu\left(a^{\prime}\right)=1$. We note that $\mathrm{CO}(F \cup G)=\mathrm{CO}^{-}(\operatorname{supp} \mu)$ is a split face which is a consequence of our assumption.

Now suppose $K$ is a metrizable simplex, $\mu$ is a boundary measure, and $F=$ $\mathrm{CO}^{-}(\operatorname{supp} \mu)$ is a face. Since $|\mu|(\partial K \cap \operatorname{supp} \mu)=1, F=\mathrm{CO}^{-}(\operatorname{supp} \mu)=\mathrm{CO}^{-}(\partial K$ $\cap \operatorname{supp} \mu)$. Note that, by Milman's theorem, $\partial F \subset \operatorname{supp} \mu$, and since $\partial K \cap \operatorname{supp} \mu \subset$ $\partial F,|\mu|(\partial F)=1$. Thus supp $\mu \subset(\partial F)^{-}$. Hence $\operatorname{supp} \mu=(\partial F)^{-}$. Also as $F$ is again a Choquet simplex, we may assume without loss of generality that $(\partial K)^{-}=$ supp $\mu$.

Let $1=\|a\|=\mu(a)$. As in the case of continuous functions, suppose $\operatorname{supp} \mu=$ $A \cup B$ for closed disjoint sets $A, B$ which is a Hahn decomposition. Suppose $A=\{x: a(x)=1\}$. We will show that $A$ is a dilated set, that is, for any boundary measure $v$ with its resultant $\gamma(v) \in A, v(A)=1$. It would then follow from a result of [3] that $\mathrm{CO}^{-}(A)$ is a face, and hence, a split face since $K$ is a simplex.

Let $v$ be a maximal measure with $\gamma(v) \in A$. Then $1=a(\gamma(v))=\int a d v$. Since $v$ is a maximal measure, $v(\{x \in \partial K: a(x)=1\})=1$. Thus $v(A)=1$.

REMARK 1.5. For a metrizable $K$ and a boundary measure $\mu$ since $|\mu|(\partial K \cap$ $\operatorname{supp} \mu)=1$, we have that $\mathrm{CO}^{-}(\operatorname{supp} \mu)=\mathrm{CO}^{-}(\operatorname{supp} \mu \cap \partial K)$. Thus if $F$ is a closed split face such that $\partial F=\operatorname{supp} \mu \cap \partial K$, then $F=\mathrm{CO}^{-}(\operatorname{supp} \mu)$. Thus in the metrizable case, for a boundary measure $\mu$, the above result can be formulated in terms of the structure topology on $\partial K$ (see [1, Chapter II, Section 6]) as supp $\mu \cap \partial K, A \cap \partial K$, and $B \cap \partial K$ are structurally closed sets. Since when $\partial K$ is closed, the structure and usual topology coincide on $\partial K$, these assumptions are consistent with the theorem of Garkavi.

We conclude with a partial result on proximinal subspaces of finite codimension of $A(K)$.

Proposition 1.6. Let $K$ be a Choquet simplex. Let $Y \subset C(K)$ be a proximinal subspace of codimension $n$ and suppose $Y^{\perp}=\operatorname{span}\left\{\mu_{1}, \ldots, \mu_{n}\right\}$ such that $E_{i}=$ $\operatorname{supp} \mu_{i} \subset \partial K$ for all $i$. Then $Y^{\prime}=\left\{a \in A(K): \mu_{i}(a)=0\right.$ for $\left.1 \leq i \leq n\right\}$ is $a$ proximinal subspace of $A(K)$.

Proof. Let $E=\cup E_{i}$. By applying Garkavi's theorem, it is easy to see that $Y^{\sim}=\left\{g \in C(E): \mu_{i}(g)=0\right.$ for $\left.1 \leq i \leq n\right\}$ is a proximinal subspace of $C(E)$. Let $a \in A(K)$ and let $f \in Y^{\sim}$ be a best approximation for $a \mid E$. Let $h \in A(K)$ 
be such that $h=a-f$ on $E$ and $\|h\|=\|a-f\|_{E}$ (see Remark 1.3). Note that, for any $b \in Y^{\prime}$, we have $b \mid E \in Y^{\sim}$. Now, for any $b \in Y^{\prime},\|a-b\| \geq\|a-b\|_{E} \geq$ $\|(a-f)\|_{E}=\|h\|=\|a-(a-h)\|$. Also $a-h \in Y^{\prime}$. Therefore, $Y^{\prime}$ is a proximinal subspace.

ACKNOWLEDGMent. This work was supported by IFCPAR Grant 2601-1.

\section{REFERENCES}

[1] E. M. Alfsen, Compact Convex Sets and Boundary Integrals, Ergebnisse der Mathematik und ihrer Grenzgebiete, vol. 57, Springer-Verlag, New York, 1971.

[2] F. Centrone and A. Martellotti, Proximinal subspaces of $C(Q)$ of finite codimension, J. Approx. Theory 101 (1999), no. 1, 78-91.

[3] E. G. Effros, Structure in simplexes. II, J. Functional Analysis 1 (1967), 379-391.

[4] R. R. Phelps, Lectures on Choquet's Theorem, 2nd ed., Lecture Notes in Mathematics, vol. 1757, Springer-Verlag, Berlin, 2001.

[5] I. Singer, Best Approximation in Normed Linear Spaces by Elements of Linear Subspaces, Die Grundlehren der Mathematischen Wissenschaften, vol. 171, Publishing House of the Academy of the Socialist Republic of Romania, Bucharest, 1970.

[6] L. P. Vlasov, On existence theorems for finite-codimensional subspaces in $C(Q)^{1}, \mathrm{~J}$. Approx. Theory 117 (2002), no. 1, 185-188.

T. S. S. R. K. Rao: Statistics and Mathematics Unit, Indian Statistical Institute, RV College Post Office, Bangalore 560 059, India

E-mail address: tss@isibang.ac.in 


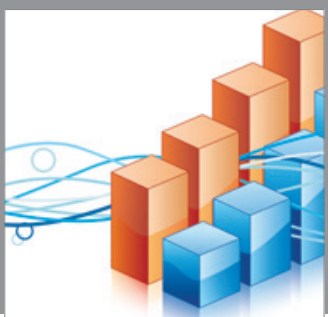

Advances in

Operations Research

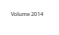

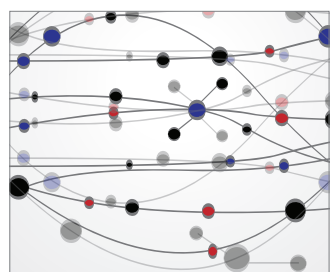

\section{The Scientific} World Journal
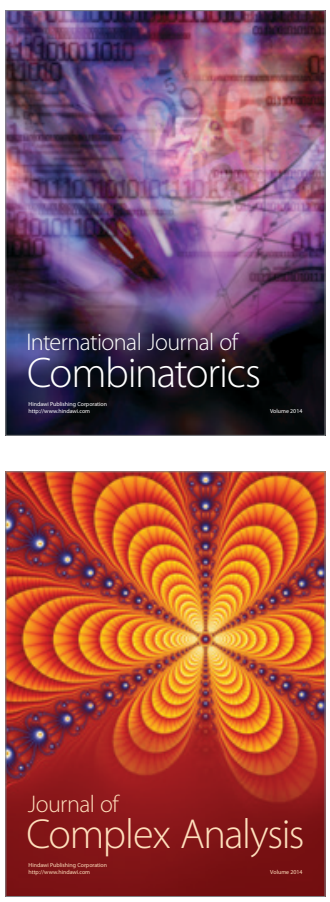

International Journal of

Mathematics and

Mathematical

Sciences
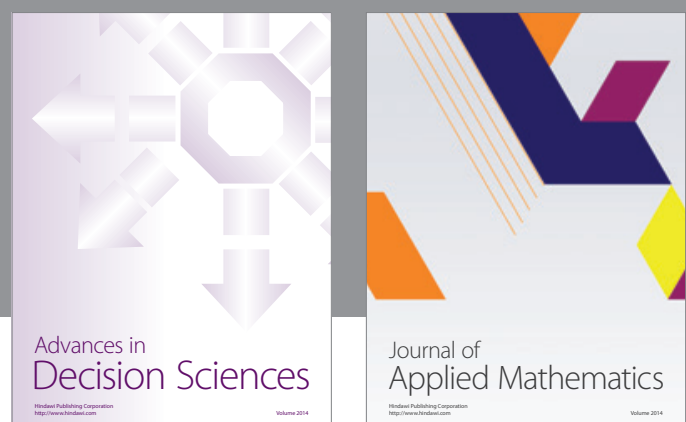

Journal of

Applied Mathematics
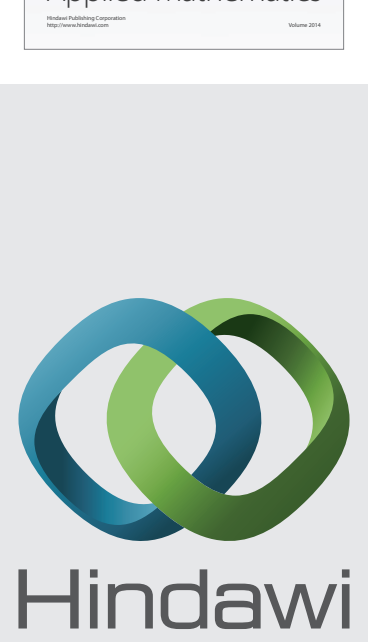

Submit your manuscripts at http://www.hindawi.com
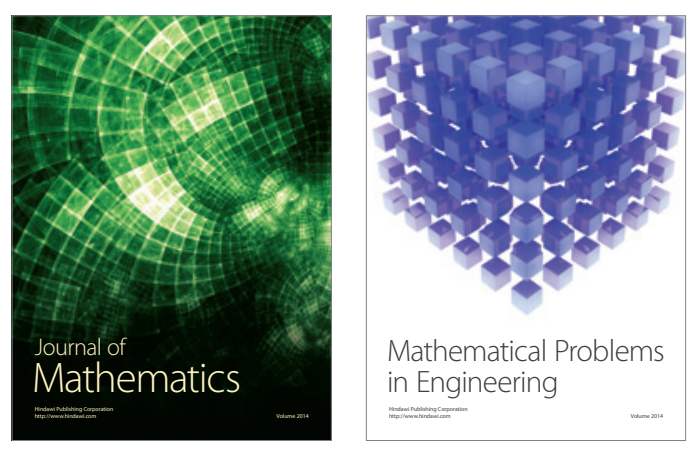

Mathematical Problems in Engineering
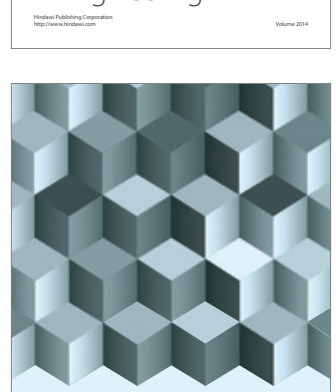

Journal of

Function Spaces
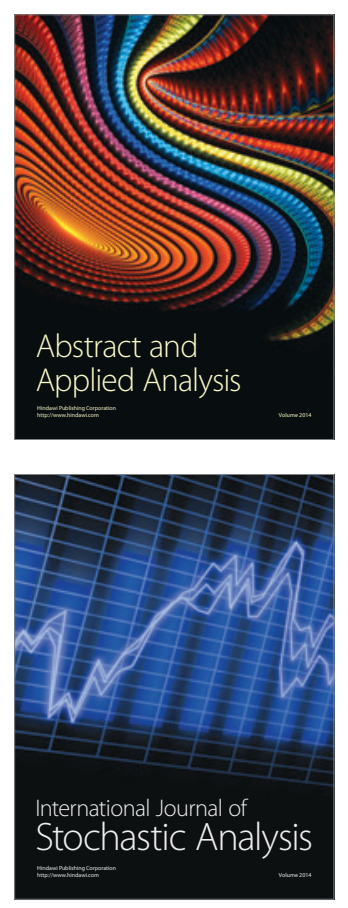

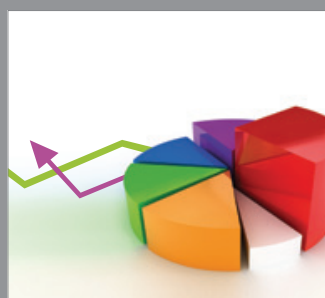

ournal of

Probability and Statistics

Promensencen
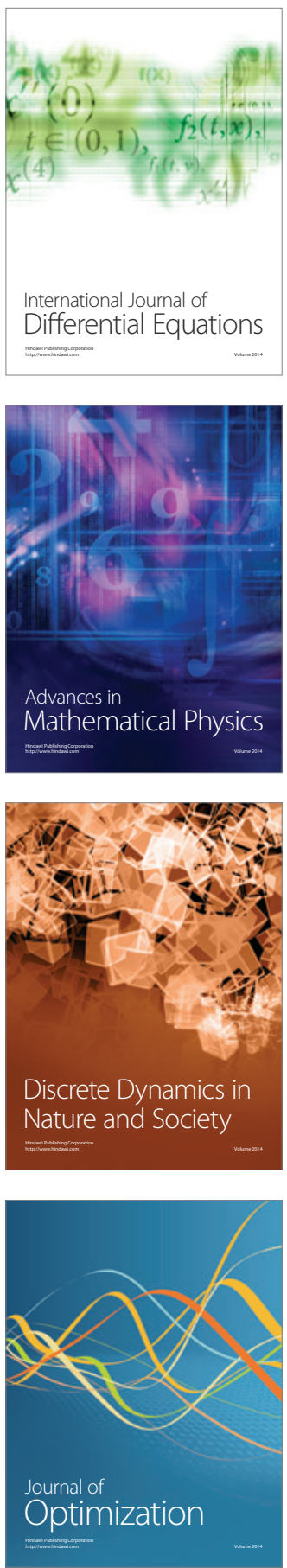